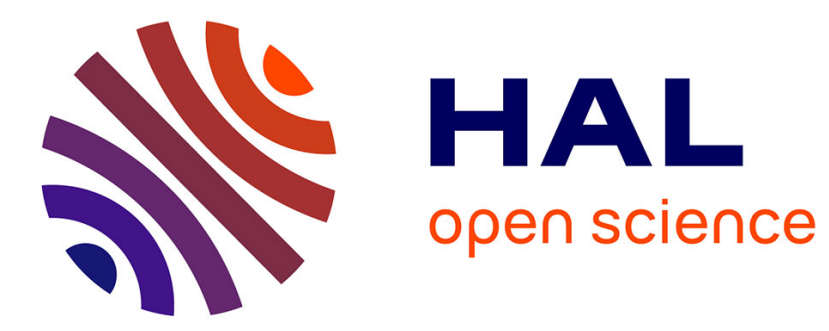

\title{
Height of convective layer in planetary atmospheres with condensable and non-condensable greenhouse substances
}

\author{
A. M. Makarieva, V. G. Gorshkov, T. Pujol
}

\section{To cite this version:}

A. M. Makarieva, V. G. Gorshkov, T. Pujol. Height of convective layer in planetary atmospheres with condensable and non-condensable greenhouse substances. Atmospheric Chemistry and Physics Discussions, 2003, 3 (6), pp.6701-6720. hal-00301398

\section{HAL Id: hal-00301398 \\ https://hal.science/hal-00301398}

Submitted on 16 Dec 2003

HAL is a multi-disciplinary open access archive for the deposit and dissemination of scientific research documents, whether they are published or not. The documents may come from teaching and research institutions in France or abroad, or from public or private research centers.
L'archive ouverte pluridisciplinaire HAL, est destinée au dépôt et à la diffusion de documents scientifiques de niveau recherche, publiés ou non, émanant des établissements d'enseignement et de recherche français ou étrangers, des laboratoires publics ou privés. 
Height of convection

A. M. Makarieva et al.

\section{Height of convective layer in planetary atmospheres with condensable and non-condensable greenhouse substances}

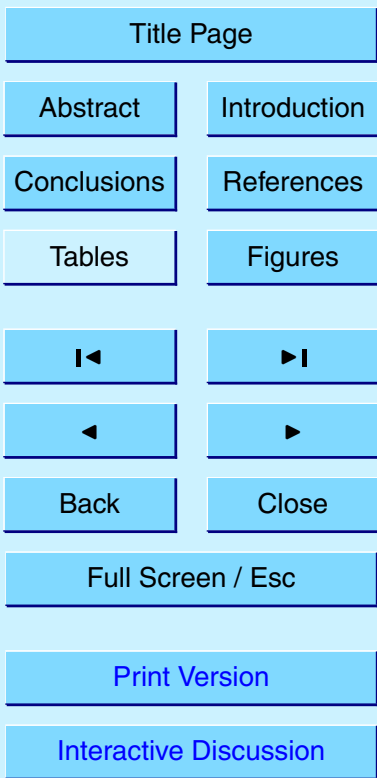

A. M. Makarieva ${ }^{1}$, V. G. Gorshkov ${ }^{1}$, and T. Pujol ${ }^{2}$

${ }^{1}$ Theoretical Physics Division, Petersburg Nuclear Physics Institute, Gatchina, St.-Petersburg, Russia

${ }^{2}$ Department of Physics, University of Girona, Girona, Catalonia, Spain

Received: 25 October 2003 - Accepted: 8 December 2003 - Published: 16 December 2003

Correspondence to: A. M. Makarieva (elba@infopro.spb.su)

Interactive Discussion

(C) EGU 2003 


\section{Abstract}

Convection reduces greenhouse effect by transporting a certain amount of nonradiative dynamic energy to the upper atmosphere, where this energy dissipates and radiates into space without interaction with greenhouse substances in the lower atmosphere. In this paper we show that the height of the convective layer $z_{c}$ is finite and independent of atmospheric optical thickness $\tau_{s}$ at large values of the latter. We derive an analytical formula for $z_{c}$ at large values of $\tau_{s}$ for condensable and non-condensable greenhouse substances. The formula obtained yields reasonable quantitative estimates of the observed height of convective layer on Venus and at low latitudes on Earth, where atmospheric thickness of water vapor is maximum.

The dissipative power of dynamic convective processes is limited by the incoming flux of solar radiation. Height of convective layer being finite, values of optical depth at the top of the convective layer and at the mean height of convective energy dissipation increase proportionally to the atmospheric optical thickness, while the contribution of convective energy fluxes to formation of the outgoing flux of thermal radiation proportionally diminishes. As far as optical thickness of condensable greenhouse substances grows exponentially with increasing surface temperature, the obtained results lead to the conclusion that the outgoing thermal radiation into space in the presence of convection tends exponentially to zero with increasing surface temperature, instead of reaching a finite plateau as suggested by earlier radiative-convective studies.

\section{Introduction}

In radiative equilibrium, when energy is transported in the atmosphere by means of radiative processes only, the ratio of the outgoing flux of thermal radiation into space to the upward flux of thermal radiation at the Earth's surface is, in accordance with Eddington's approximation, inversely proportional to the amount of greenhouse substances in the atmosphere. The atmospheric content of the major greenhouse substance on Earth
ACPD

$3,6701-6720,2003$

Height of convection

A. M. Makarieva et al.

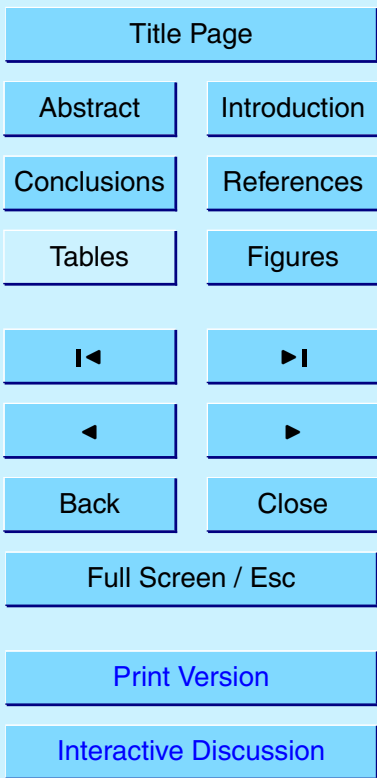

(C) EGU 2003 
- water vapor - in the presence of liquid hydrosphere rises exponentially with increasing surface temperature. With an account made for the relevant increase in cloudiness absorbing thermal radiation over entire thermal spectrum, this corresponds to an exponential decline of the outgoing flux of thermal radiation into space with increasing 5 surface temperature.

Non-radiative convective processes could transport a certain amount of dynamic energy to the upper radiative layer of the atmosphere. There this energy would dissipate and radiate directly into space, avoiding interaction with greenhouse substances in any atmospheric layers lower than the upper radiative one. If this convective contri10 bution to the flux of thermal radiation into space were independent of the amount of greenhouse substances in the atmosphere, the thermal flux into space would not diminish exponentially with surface temperature, as in the case of radiative equilibrium, but ultimately reached a non-zero plateau. Such a scenario was suggested by several radiative-convective studies (Komabayasi, 1967; Ingersoll, 1969; Kasting, 1988; 15 Goody and Yung, 1989; Nakajima et al., 1992; Rennó, 1997; Pujol and North, 2002). Such a scenario would have profoundly different implications for the thermal evolution of the planets than the exponentially diminishing thermal flux into space. It is important therefore to verify carefully the physical assumptions underlying the two scenarios.

A constant convective contribution to thermal flux into space implies that at any value 20 of atmospheric optical thickness convection propagates up to a fixed optical depth, e.g. to the upper radiatve layer of the atmosphere, which corresponds to optical depth of about unity. In this paper we demonstrate that convection propagates up to a fixed atmospheric height rather than to a fixed optical depth. The optical depth corresponding to the top of the convective layer grows proportionally to atmospheric optical thickness.

25 Given that the dissipative power of the dynamic convective processes is limited by the incoming solar flux, this means that the convective contribution to thermal flux into space diminishes inversely proportionally to the growing atmospheric optical thickness and does not reach a finite plateau at high surface temperatures. This result allows to conclude that at large values of atmospheric optical thickness the condition of ra-
ACPD

3, 6701-6720, 2003

Height of convection

A. M. Makarieva et al.

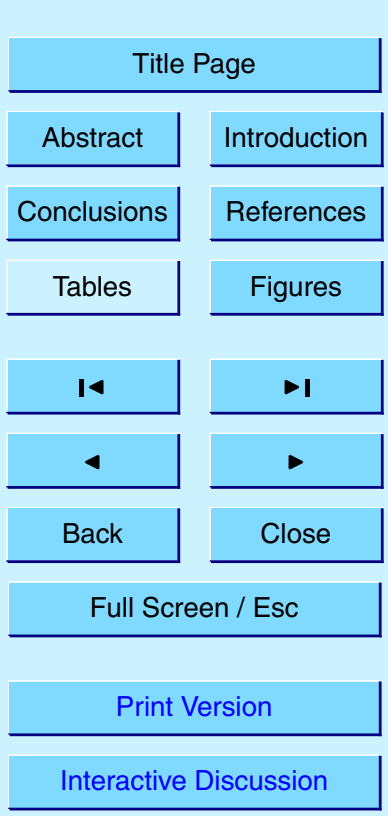

(C) EGU 2003 
diative equilibrium described by Eddington's approximation is fulfilled not only in the stratosphere, but spreads to the entire atmosphere.

In Sect. 2 we consider how height of convective layer changes with increasing opACPD tical thickness of an atmosphere containing non-condensable greenhouse gases only 5 (like $\mathrm{CO}_{2}$ ). We show that the height of convective layer is finite and independent of atmospheric optical thickness at large values of the latter. We demonstrate that the developed theoretical approach yields the exact numerical value for the height of convective layer on Venus.

In Sect. 3.1 we extend our consideration to condensable greenhouse substances 10 and show that the results obtained are valid for this case as well. We estimate the maximum height of convective layer in an atmosphere saturated with water vapor. The obtained estimate displays a good agreement with the observed height of convection at low latitudes on Earth, where atmospheric content of water vapor is maximum.

In Sect. 3.2 we show that the presence of convection in the terrestrial atmosphere 15 does not decelerate the exponential decline of the outgoing flux of thermal radiation into space with growing surface temperature.

\section{Convection in an atmosphere with non-condensable greenhouse substances}

\subsection{Brightness temperature}

In the state of radiative equilibrium the dependence between the upward fluxes of ther-

mal radiation into space, $F_{e}$, and at optical depth $\tau$ in the atmosphere, $F^{+}(\tau)$, in a spectral interval of resonance absorption of thermal radiation by atmospheric greenhouse substances is accurately described by Eddington's approximation (Michalas and Michalas, 1984):

$F^{+}(\tau)=F_{e}(1+k \tau), \quad k=\frac{3}{4} ; \quad F_{s}^{+}=F_{e}\left(1+k \tau_{s}\right)$

Height of convection

A. M. Makarieva et al.

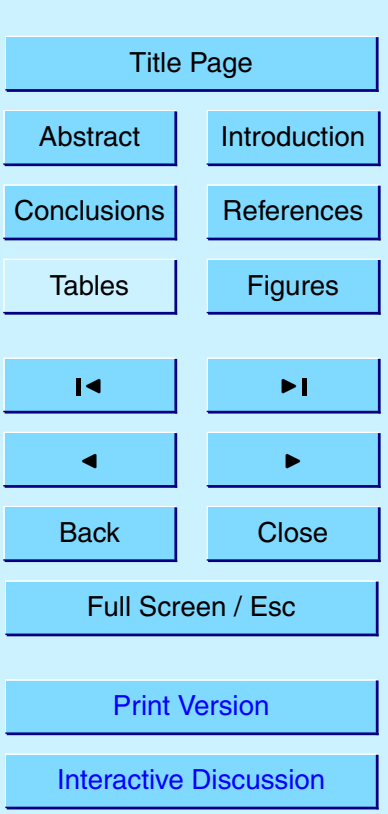

(C) EGU 2003 
Here and below low index $s$ refer to vales at the Earth's surface. At the Earth's surface the upward flux of thermal radiation is related to surface temperature $T_{s}$ by StephenBoltzmann formula for the black body radiation. For any optical depth $\tau$ (and corresponding height $z$ ) the so-called brightness temperature $T_{b}$ in a given spectral interval ${ }_{5} \Delta \lambda \equiv \lambda_{2}-\lambda_{1}$ is defined as follows:

$F^{+}(\tau)=\int_{\lambda_{1}}^{\lambda_{2}} I_{P}\left(\lambda, T_{b}\right) d \lambda \equiv \delta_{\Delta \lambda} \sigma T_{b}^{4}, \quad F_{s}^{+}=\delta_{\Delta \lambda} \sigma T_{s}^{4}$.

Here $T_{s}$ is the surface temperature, $\sigma$ is the Stephen-Boltzmann constant, $I_{\mathrm{P}}\left(\lambda, T_{b}\right)$ is Planck's function, $\delta_{\Delta \lambda}$ is the relative share of the observed flux $F^{+}(\tau)$ of thermal radiation within spectral interval $\Delta \lambda$ in the total flux of black body radiation of temperature ${ }_{10} T_{b}$. We assume $\delta_{\Delta \lambda}$ to be independent of $T_{b}$ and height $z$. This assumption is valid for a grey greenhouse substance (e.g. clouds or gas above the critical point) for which $\delta_{\Delta \lambda} \sim 1$. It is also valid when brightness temperature in the interval $\Delta \lambda$ changes relatively little within the convective layer.

Using Eqs. (2.1) and (2.2) we obtain the following expression of brightness temper15 ature $T_{b} \equiv T_{b}(z)$ in terms of optical depth $\tau \equiv \tau(z)$ :

$T_{b}=T_{s}\left(\frac{1+k \tau}{1+k \tau_{s}}\right)^{1 / 4}$

Brightness temperature is maximum at the Earth's surface and decreases monotonically with growing height $z$ due to the monotonical decrease of $\tau$ with height $z$. The lapse rate of brightness temperature $G_{b} \equiv-d T_{b} / d z$ also monotonically decreases 20 with height.

We note that at any height $z>0$ the local air temperature $T$ is higher than the brightness temperature $T_{b}, T(z)>T_{b}(z)$. This follows from the direction of energy conversion: the energy of non-radiative fluxes which, upon its dissipation, is characterized by air temperature $T$, is converted to the energy of the excited levels of molecules of

ACPD

$3,6701-6720,2003$

Height of convection

A. M. Makarieva et al.

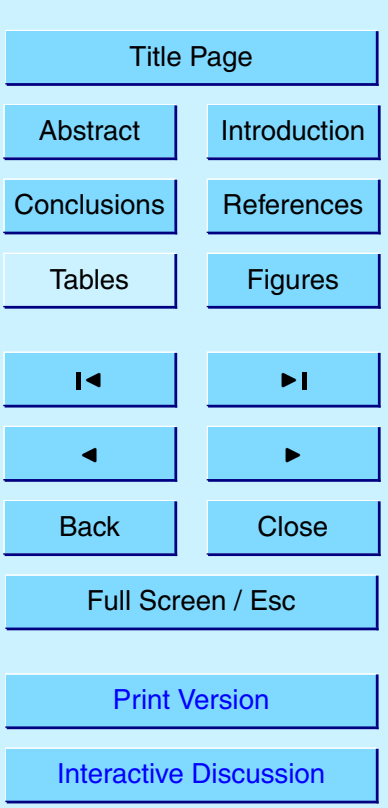

(C) EGU 2003 
greenhouse substances with subsequent emission of thermal radiation characterized by brightness temperature $T_{b}$. The relation $T>T_{b}$ for $z>0$ and the monotonical smooth decline of both $T$ and $T_{b}$ with height mean that the lapse rate of air temperature $G \equiv-d T / d z$ is always lower than the lapse rate of brightness temperature ${ }_{5} G_{b} \equiv-d T_{b} / d z$.

\subsection{Temperature lapse rate and the onset of convection}

The gaseous atmosphere in the gravitational field of Earth conforms to the equation of hydrostatic equilibrium, $-d p / d z=M N g$, and the equation of state for atmospheric air, $p=N R T$, where $p, N$ and $T$ are pressure, molar concentration and temperature of air 10 at with observations, $R=8.3 \mathrm{~J} \mathrm{~mole}^{-1} \mathrm{~K}^{-1}$ is gas constant, $g$ is acceleration of gravity. The two equations can be combined in the following well-known form:

$\frac{d p}{d z}=-\frac{p}{h} ; \quad h \equiv \frac{R T}{M g}$.

Relation (2.4) is valid for all gases with constant mass fraction, in particular, for noncondensable greenhouse substances like $\mathrm{CO}_{2}$. Due to the observed constant molar mass of air, the ratio $p / p_{s}$ in Eq. (2.4) is, as is well-known, equal to the corresponding ratio of partial pressures of such gases and to the ratio $\tau / \tau_{s}$, i.e. $p / p_{s}=\tau / \tau_{s}$. Using this equality together with Eqs. (2.3) and (2.4) we obtain:

$$
\begin{aligned}
& G_{b} \equiv-\frac{d T_{b}}{d z}=G_{\max } \alpha_{b}, \quad G_{\max } \equiv \frac{T_{s}}{4 h_{s}}=\frac{M g}{4 R}, \\
& \alpha_{b}=\frac{k \tau}{(1+k \tau)^{3 / 4}\left(1+k \tau_{s}\right)^{1 / 4}} \cdot \frac{T_{s}}{T} .
\end{aligned}
$$

20 Here $h_{s}$ is the scale height of distribution of non-condensable gases in the atmosphere. The fundamental value of $G_{\max }$ is a convenient scale for representing any temperature lapse rates $G_{i}$ in the form of dimensionless variables $\alpha_{i} \equiv G_{i} / G_{\max }$.

\section{6}

ACPD

3, 6701-6720, 2003

Height of convection

A. M. Makarieva et al.

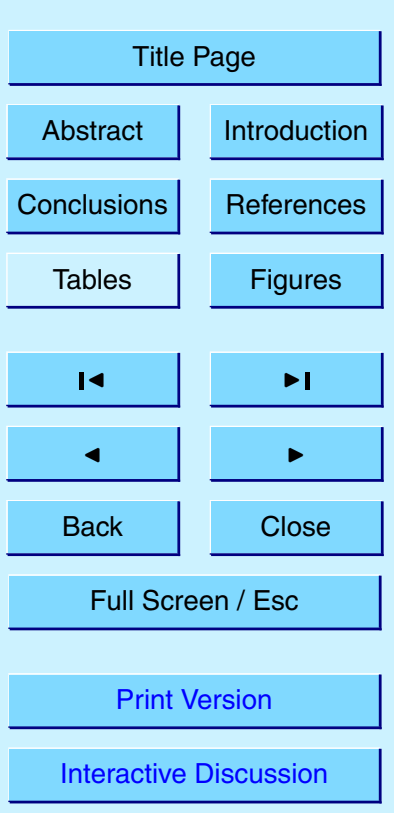

(C) EGU 2003 
Convective processes in the atmosphere set in when the lapse rate of air temperature exceeds some critical value $\Gamma$ (Chandrasekhar, 1957; Ramanathan and Coakley, 1978; Weaver and Ramanathan, 1995). In the terrestrial atmosphere $G_{\max }=$ $8.56 \mathrm{~K} \mathrm{~km}^{-1}$, while convection exists at the observed lapse rate $\Gamma=6.5 \mathrm{~K} \mathrm{~km}^{-1}$. This 5 value can be viewed as the critical lapse rate for the advent of convection in the terrestrial atmosphere (Ramanathan and Coakley, 1978). The condition $\Gamma<G_{\max }$ implied by Eq. (2.5) is therefore fulfilled in the terrestrial atmosphere.

With increasing amount of greenhouse substances in the atmosphere the lapse rate of brightness temperature starts to grow, first of all at the surface, as far as $G_{b s}>G_{b}(z)$ 10 for any $z>0$. At some value of atmospheric optical thickness $\tau_{s}=\tau_{s \text { min }}$ the lapse rate of brightness temperature at the surface may reach the critical value, $G_{b s}=\Gamma$, so that it becomes possible for convection to originate. It happens if the lapse rate of air temperature follows $G_{b}$ closely enough. With increasing $\tau_{s}>\tau_{s \text { min }}$ the convective layer spreads upwards till yet larger and larger heights.

15 Once convection sets in, it works to keep the lapse rate of air temperature $G$ close to the critical value of $\Gamma$ (Ramanathan and Coakley, 1978). Thus, within the convective layer $z \leq z_{C}$ the lapse rate of air temperature is approximately constant, $G \approx \Gamma$, and air temperature obeys the observed relation:

$T=T_{s}-\Gamma z=T_{s}\left(1-\frac{\alpha}{4} \frac{z}{h_{s}}\right), \quad \alpha \equiv \frac{\Gamma}{G_{\max }}$.

20 For Earth $\Gamma=6.5 \mathrm{~K} \mathrm{~km}^{-1}$ and $\alpha=0.77$. At $z>z_{C}$ there is no convection, and Eq. (2.6), as well as any of its derivations, may not be used for description of air temperature.

Using Eqs. (2.4) and (2.6) we obtain the following well-known relation for $z \leq z_{C}$ :

$\frac{\tau}{\tau_{s}}=\frac{p}{p_{s}}=\left(1-\frac{\alpha}{4} \frac{z}{h_{s}}\right)^{4 / \alpha}$.

Convection exists in that part of the atmosphere where air temperature lapse rate $25 G$ exceeds the critical value $\Gamma, G>\Gamma$. As far as $G<G_{b}$, the maximum height $z_{c}$ of 6707
ACPD

3, 6701-6720, 2003

Height of convection

A. M. Makarieva et al.

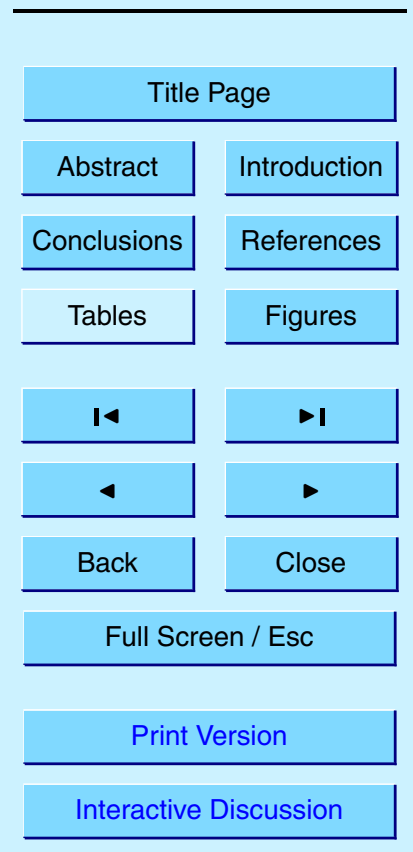

(C) EGU 2003 
convective layer at a given value of optical thickness $\tau_{s}$ and surface temperature $T_{s}$ can be obtained from the equation $G_{b}\left(z_{c}\right)=\Gamma$ or, using Eqs. (2.5) and (2.6), from the equation

$\alpha_{b}=\alpha$.

5 Variables $\tau$ and $T$ in Eq. (2.8) are related to height $z$ by Eqs. (2.7) and (2.6), respectively. Equation (2.8) determines the maximum height of the convective layer $z=z_{C}$ at different values of atmospheric optical thickness $\tau_{s}$, Fig. 1 .

The threshold atmospheric optical thickness $\tau_{s \text { min }}$ is obtained from Eq. (2.8) by putting $z=0$, which means $\tau=\tau_{s}$ and $T=T_{s}$ :

${ }_{10} \tau_{s \min }=\frac{1}{k} \frac{\alpha}{1-\alpha}$.

For Earth we obtain $\tau_{s \text { min }}=4.5$. The major non-condensable greenhouse substance in the terrestrial atmosphere is $\mathrm{CO}_{2}$. Atmospheric optical thickness of $\mathrm{CO}_{2}$ in the $15 \mu \mathrm{m}$ spectral interval is estimated as $k \tau_{s} \mathrm{CO}_{2} \approx 1.9$ (Gorshkov and Makarieva, 2002), which corresponds to $\tau_{s \mathrm{CO}_{2}}=2.5$ at $k=3 / 4$. Thus, the atmospheric $\mathrm{CO}_{2}$ concentration on Earth is insufficient for switching convection on. As we show in the next section, convection on Earth is switched on by atmospheric water vapor. On Venus, on the contrary, atmospheric $\mathrm{CO}_{2}$ concentration appears to be by far sufficient for convection to originate, see below.

2.3. Height of convective layer at large values of $\tau_{s}$

20 As one can see from Fig. 1, the height of convective layer $z_{C}$ reaches a finite limit at large values of atmospheric optical thickness. For $\tau_{s} \rightarrow \infty\left(\tau_{s} \gg 1\right)$ one can use Eqs. (2.5)-(2.7) to re-write Eq. (2.8) as

$$
\left(\frac{p}{p_{s}}\right)^{1 / 4} \frac{T_{s}}{T}=\alpha .
$$

\section{Height of convection}

A. M. Makarieva et al.

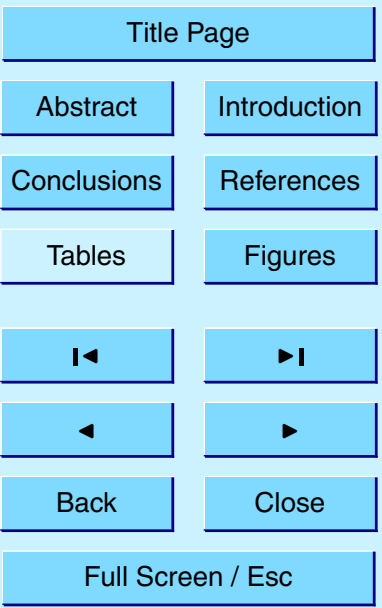

Print Version

Interactive Discussion

(C) EGU 2003 
Putting Eqs. (2.6) and (2.7) into Eq. (2.10) we obtain an exact finite value for the height of the convective layer $z_{c}$ max at $\tau_{s} \rightarrow \infty$ :

$\frac{z_{c \max }}{h_{s}}=4 \frac{1}{\alpha}\left(1-\alpha^{\alpha /(1-\alpha)}\right)=3.03$.

For modern Earth we have $T_{s}=288 \mathrm{~K}, h_{s}=8.4 \mathrm{~km}$ and $z_{c \text { max }}=25 \mathrm{~km}$. Eq. (2.11) 5 describes the maximum height of the convective layer that could form in the terrestrial atmosphere with non-condensable greenhouse substances as the only absorbers of thermal radiation.

Minimum values of air temperature $T_{c} \equiv T\left(z_{c}\right)$ and brightness temperature $T_{b c} \equiv$ $T_{b}\left(z_{c}\right)$ at the top of the convective layer, $z_{c}=z_{c \max }$, at large values of atmospheric 10 optical thickness, $\tau_{s} \gg 1$, are obtained by substituting Eq. (2.11) into Eq. (2.6), and Eq. (2.11) into Eqs. (2.7) and (2.3), respectively:

$$
\begin{aligned}
& \frac{T_{c}}{T_{s}}=\alpha^{\alpha /(1-\alpha)}, \quad \frac{T_{b c}}{T_{s}}=\alpha^{1 /(1-\alpha)} ; \\
& \frac{T_{c}}{T_{s}}=0.42, \quad \frac{T_{b c}}{T_{s}}=0.32, \quad \alpha=0.77 .
\end{aligned}
$$

The numeric values in the second line of Eq. (2.12) correspond to modern Earth.

As is clear from Eq. (2.12), brightness temperature $T_{b c}$ at the top of the convec15 tive layer grows proportionally to surface temperature $T_{s}$. Upward flux $F_{c}^{+}$of thermal radiation at the top of the convective layer increases therefore proportionally to $T_{s}^{4}$. The dissipative power of non-radiative convective fluxes of energy and, consequently, collisional excitation of the absorption bands of greenhouse substances, are limited by the incoming flux of solar radiation $F_{\text {in }}$, which does not change with increasing $\tau_{s}$ 20 or $T_{S}$. (We note that in the non-stationary case the absorbed flux of solar radiation $F_{\text {in }}$ is not equal to the outgoing flux of thermal radiation $F_{e}, F_{\text {in }} \neq F_{e}$, and also that $F_{s}^{+} / F_{\text {in }} \neq 1+k \tau_{s}$.) When the upward flux of thermal radiation at the surface is much larger than the absorbed flux of solar radiation, $F_{s}^{+}=\sigma T_{s}^{4} \gg F_{\text {in }}$, convective processes 6709

\section{Height of convection}

A. M. Makarieva et al.

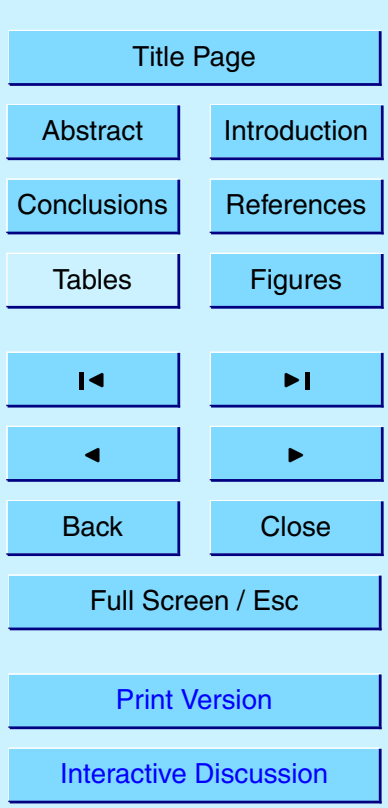

(C) EGU 2003 
are unable to significantly perturb the upward fluxes of thermal radiation and brightness temperature within the convective layer as compared to the case of radiative equilibrium. With growing $\tau_{s}$ and $T_{s}$ the radiative equilibrium spreads from the stratosphere to the entire atmosphere, so that Eddington's approximation becomes valid for the entire 5 atmosphere as well. Therefore, Eqs. (2.11) and (2.12) obtained with use of Eddington's approximation yield at $F_{s}^{+} \gg F_{\text {in }}$ and $\tau_{s} \gg 1$ the exact values of $z_{c}, T_{b c}$ and $T_{c}$.

On Venus the ratio of the upward flux of thermal radiation at the surface $F_{s}^{+}$to the outgoing flux of thermal radiation into space $F_{e}=F_{\text {in }}$ equals $F_{s}^{+} / F_{e}=98$ (Mitchell, 1989). In accordance to Eddington's approximation (2.1) this corresponds to $\tau_{s}=$ $10 \frac{4}{3}\left(F_{s}^{+} / F_{e}\right)=131 \gg 1$. Thus, Eqs. (2.11) and (2.12) can be applied to Venus with a high accuracy. Taking for Venus $h_{s}=15.6 \mathrm{~km}, G_{\max }=11.4 \mathrm{~K} \mathrm{~km}^{-1}\left(T_{s}=730 \mathrm{~K}, M_{\mathrm{CO}_{2}}=\right.$ $44 \mathrm{~g} \mathrm{~mole}^{-1}, g=8.6 \mathrm{~m} \mathrm{~s}^{-2}$ (Allen, 1973; Kasting, 1988; Mitchell, 1989)), the observed air temperature lapse rate $\Gamma \approx 8 \mathrm{~K} \mathrm{~km}^{-1}$ (Goody and Yung, 1989), we obtain for Venus $\alpha \equiv \Gamma / G_{\max }=0.70$. Putting these values into Eq. (2.11), we obtain the following 15 values for the height $z_{c \text { max }}$ of the convective layer on Venus, $z_{c \text { max }}=50.4 \mathrm{~km}$. This value agrees very well with the observed height of cloudiness on Venus ( $50 \mathrm{~km})$, where, according to observations (Goody and Yung, 1989), the air temperature lapse rate starts to diminish becoming less than the critical convective value. From (2.12) we calculate air temperature at $z_{c \text { max }}$ for Venus to be $T_{c}=318 \mathrm{~K}\left(44^{\circ} \mathrm{C}\right)$.

We note that the height of convection $z_{c}$ is generally less than the height of the tropopause $z_{t p p}, z_{c}<z_{t p p}$. The latter is usually defined as the point where the lapse rate of air temperature approaches zero. As suggested by Fig. 1, the lapse rate of brightness temperature and, consequently, lapse rate of air temperature may remain substantial for $z>z_{c}$, being nevertheless smaller than the critical value $\Gamma$ responsible
ACPD

$3,6701-6720,2003$

Height of convection

A. M. Makarieva et al.

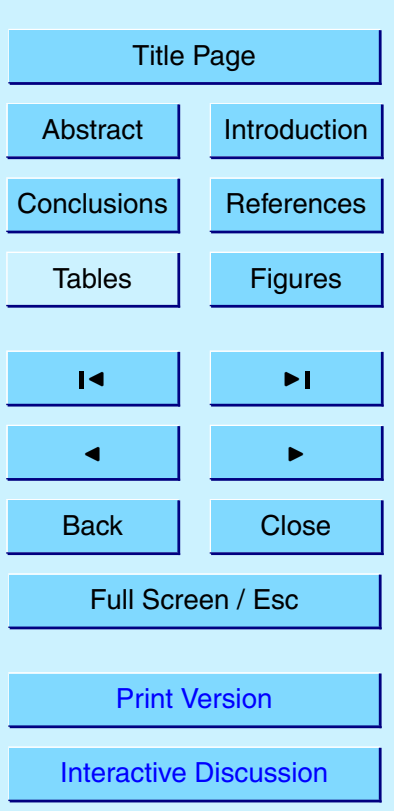

(C) EGU 2003 


\section{Convection in an atmosphere with condensable greenhouse substances}

3.1. Height of convective layer

$3,6701-6720,2003$

The dependence of saturated water vapor pressure $p_{L}$ on temperature is described by Clausius-Clapeyron equation (everywhere below the low index $L$ denotes variables 5 corresponding to water vapor):

$p_{L}=p_{s L} \exp \left(\frac{T_{L}}{T_{s}}-\frac{T_{L}}{T}\right), \quad T_{L} \equiv \frac{Q}{R} \approx 5300 \mathrm{~K}$,

where $Q \approx 44 \mathrm{KJ} \mathrm{mole}^{-1}$ is the latent heat of evaporation of water vapor, $p_{s L}$ is partial pressure of water vapor at the surface.

In an isothermal atmosphere water vapor can be saturated only immediately above 10 the water surface, $z=0$. In the gravitational field the concentration of water vapor drops exponentially with height, so that at $z>0$ water vapor becomes unsaturated.

If we demand that water vapor in the atmosphere is saturated and is in hydrostatic equilibrium at all heights $z$, the corresponding lapse rate $\Gamma_{L}$ of air temperature is obtained from the condition that both Eqs. (2.4) and (3.1) hold:

$\Gamma_{L} \equiv-\frac{d T}{d z}=\frac{T_{s}}{H_{L}} \exp \left(-\frac{z}{H_{L}}\right) \approx \frac{T_{s}}{H_{L}}$,

$H_{L} \equiv \frac{R T_{L}}{g M}=154 \mathrm{~km}$.

Due to the large value of $H_{L}$ Eq. (3.1), for a relatively wide interval of surface temperature changes $\Gamma_{L}$ is practically constant (independent of $z$ ) in the entire troposphere.

If the lapse rate of air temperature $G$ were smaller than $\Gamma_{L}$, atmospheric water vapor would be unsaturated at all heights $z$.

20 In the modern atmosphere of Earth we have

Height of convection

A. M. Makarieva et al.

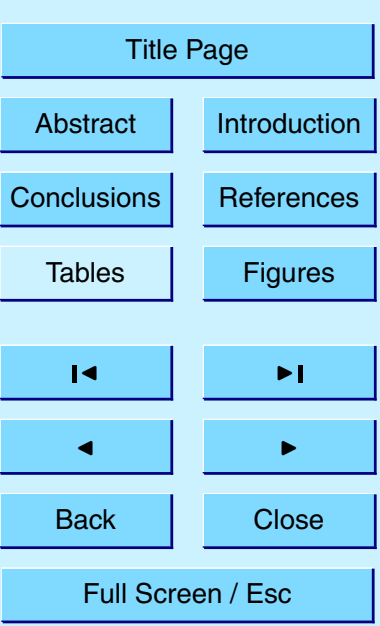

$G \approx \Gamma=\beta_{s} \Gamma_{L}, \quad \beta_{s}=\frac{\alpha H_{L}}{4 h_{s}}=\alpha \frac{T_{L}}{4 T_{s}}=3.5$.

Print Version

Interactive Discussion

(C) EGU 2003 
As far as the observed lapse rate $G \approx \Gamma$ of air temperature is steeper than $\Gamma_{L}$, $\Gamma>\Gamma_{L}$, the distribution of water vapor in the atmosphere differs from the hydrostatic equilibrium distribution (2.4). Calculating $d p_{L} / d z$ with use of Eqs. (3.1) and (2.4) we find for the convective layer $z<z_{C L}$ :

${ }_{5} \frac{d p_{L}}{d z}=-\frac{p_{L}}{h_{L}}, \quad h_{L}=\frac{h}{\beta}, \quad \beta \equiv \alpha \frac{T_{L}}{4 T} \approx \beta_{s}=3.5$.

As is clear from Eq. (3.4), the modern distribution of water vapor in the atmosphere is compressed by $\beta \approx 3.5$ times as compared to distribution of air (Weaver and Ramanathan, 1995). The scale height of water vapor distribution in the modern atmosphere equals $h_{s L} \equiv h_{s} / \beta_{s} \approx 2.4 \mathrm{~km}$, which agrees well with observations (Goody and

10 Yung, 1989; Weaver and Ramanathan, 1995). Compression of water vapor in the modern atmosphere significantly violates hydrostatic equilibrium and brings about intensive dynamic fluxes of hydrosphere evaporation, upward transport of evaporated water and compensating downward fluxes of precipitation.

Assuming approximate constancy of $\beta$ in Eq. (3.4) we obtain an approximate relation similar to Eq. (2.7):

$$
\begin{aligned}
\frac{\tau_{L}}{\tau_{s L}} \approx \frac{p_{L}}{p_{s L}} \approx\left(1-\frac{\alpha_{L}}{4} \frac{z}{h_{s L}}\right)^{4 / \alpha_{L}}= & \left(\frac{p}{p_{s}}\right)^{\beta_{s}}, \quad z<z_{c}, \\
& h_{s L} \equiv \frac{h_{s}}{\beta_{s}}, \quad \alpha_{L} \equiv \frac{\alpha}{\beta_{s}} .
\end{aligned}
$$

Due to the large value of $\beta_{s}$ and the rapid drop of pressure with height, relation Eq. (3.5) differs from Eq. (3.1) for the area of interest $z \leq h_{s L}$ by magnitudes of the order of $\alpha_{L} / 4=T_{s} / T_{L} \sim 0.05$, i.e. by less than $5 \%$.

20 Relation (3.5) for condensable greenhouse substances appear to be identical to the corresponding relations (2.4) for non-condensable greenhouse substances if one substitutes $\alpha$ and $h_{s}$ by $\alpha_{L}$ and $h_{s L}$, respectively, the latter being $\beta_{s}$ times lower than the for-

ACPD

3, 6701-6720, 2003

Height of convection

A. M. Makarieva et al.

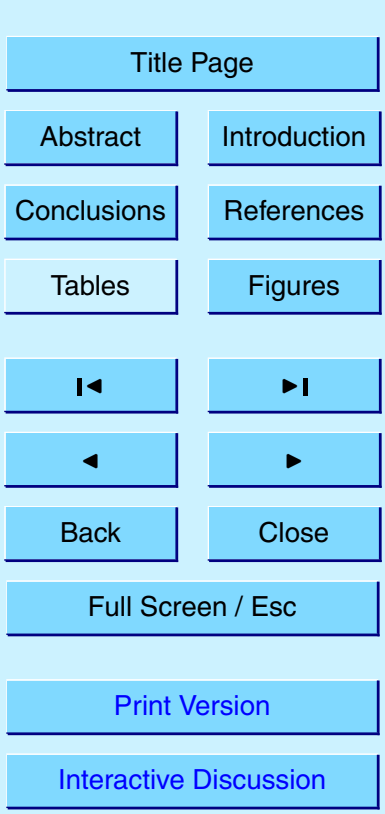

(C) EGU 2003 
mer. Similarly, the maximum value of brightness temperature lapse rate $G_{\max }=T_{s} / 4 h_{s}$ ACPD (2.5) becomes for water vapor $\beta_{s}$ times larger:

$3,6701-6720,2003$

$G_{L \max }=T_{s} / 4 h_{s L}=G_{\max } \beta_{s}$

i.e. equal to $30 \mathrm{~K} \mathrm{~km}^{-1}$ for Earth.

5 The expression for maximum height of the convective layer determined by water vapor, $z_{C L \text { max }}$, is obtained from Eq. (2.8) by a similar substitution $\alpha \rightarrow \alpha_{L}, \tau \rightarrow \tau_{L}$ and $\tau_{s} \rightarrow \tau_{s L}$, where $\alpha_{L}=\alpha / \beta_{s}=0.22, \tau_{L}$ is determined by Eq. (3.5) and temperature $T$ is determined by Eq. (2.6). The onset of convection in the terrestrial atmosphere due to the greenhouse effect of water vapor occurs at $\tau_{s L \text { min }}=0.38$, Fig. 2 , which is more 10 than ten times smaller than $\tau_{s \text { min }}=4.5$ for non-condensable greenhouse substances like $\mathrm{CO}_{2}$, Fig. 1. The atmospheric optical thickness of water vapor in the terrestrial atmosphere is estimated as $k \tau_{s L} \approx 0.53$ (Gorshkov and Makarieva, 2002), which exceeds the value of $\tau_{s L}$ min by almost twofold. This makes it possible to conclude that convection in the terrestrial atmosphere is switched on and maintained by water vapor. The idea that water vapor is more efficient with respect to switching on convection than non-condensable absorbers like $\mathrm{CO}_{2}$ due to the above discussed compression of water vapor in the modern atmosphere, was put forward by Weaver and Ramanathan (1995).

Note that in the intervals of $\tau_{s L}<\tau_{s L \text { min }}$ corresponding to $\Gamma>G>\Gamma_{L}$ water vapor is already not in hydrostatic equilibrium (i.e. there are fluxes of evaporation and precipitation), while atmospheric convection is still absent.

At large values of optical thickness $\tau_{s} \gg 1$ Eqs. (2.11) and (2.12) retain their form for water vapor if one substitutes $h_{s} \rightarrow h_{s L}=h_{s} / \beta_{s}$ and $\alpha \rightarrow \alpha_{L}=\alpha / \beta_{s}$ :

$$
\begin{aligned}
z_{c L \text { max }}=4 h_{s L} & \frac{1}{\alpha_{L}}\left(1-\alpha_{L}^{\alpha_{L} /\left(1-\alpha_{L}\right)}\right) \approx \\
& \approx 4 \frac{h_{s}}{\beta_{s}} \ln \frac{\beta_{s}}{\alpha}=6.3 \frac{h_{s}}{\beta_{s}}=15 \mathrm{~km} .
\end{aligned}
$$

\section{Height of convection}

A. M. Makarieva et al.

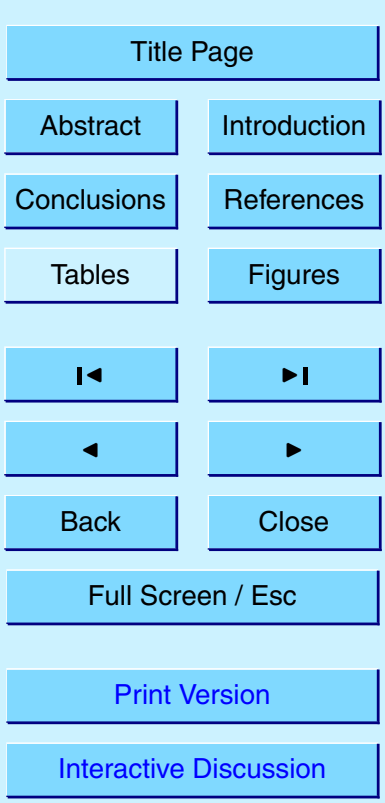

(C) EGU 2003 
The values of air temperature $T_{c L}$ and brightness temperature $T_{b c L}$ at the top of convective layer at $z=z_{C L}$ max are obtained from Eqs. (2.12) by substituting $\alpha$ for $\alpha_{L}$. At $T_{S}=288 \mathrm{~K}$ and $\alpha_{L}=0.22$ we have $T_{C L}=188 \mathrm{~K}=-85^{\circ} \mathrm{C}$.

On Earth the atmospheric content of water vapor is strongly dependent on surface 5 temperature rising approximately twofold per each ten degrees of surface temperature increase, see Eq. (3.1). The maximum values of atmospheric optical thickness of water vapor are therefore to be observed in the equatorial regions (Raval and Ramanathan, 1989). In accordance with Eq. (3.7), in these regions one also observes maximum height of the convective layer of the order of $15 \mathrm{~km}$ and minimum air temperatures of

10 the order of $-80^{\circ} \mathrm{C}$. In the polar regions where surface temperature and, consequently, atmospheric water vapor content, are much lower, one should expect to observe significantly lower convective layer and higher temperatures at its top, which is indeed the pattern observed.

\subsection{Outgoing flux of thermal radiation into space}

Clausius-Clapeyron equation (3.1) can be viewed as determining partial pressure of saturated water vapor at an arbitrary height $z$ at an arbitrary moment of time $t$. It is easy to see that Eq. (3.1) conforms to the following invariance rule:

$p_{s L} \exp \left(\frac{T_{L}}{T_{S}}\right)=p_{s L 0} \exp \left(\frac{T_{L}}{T_{s 0}}\right)$

where $T_{s 0}$ and $p_{s L 0}$ are the values of surface temperature and partial pressure at the initial moment of time $t=0$, which can be taken corresponding to the state of thermal equilibrium $F_{e}=F_{\text {in }}$. It is easy to see from Eq. (3.8) that optical thickness of atmospheric water vapor $\tau_{s L}$, as well as partial pressure of water vapor at the Earth's surface $p_{s L}$, see Eq. (3.5), increase exponentially with growing increment of surface

ACPD

$3,6701-6720,2003$

Height of convection

A. M. Makarieva et al.

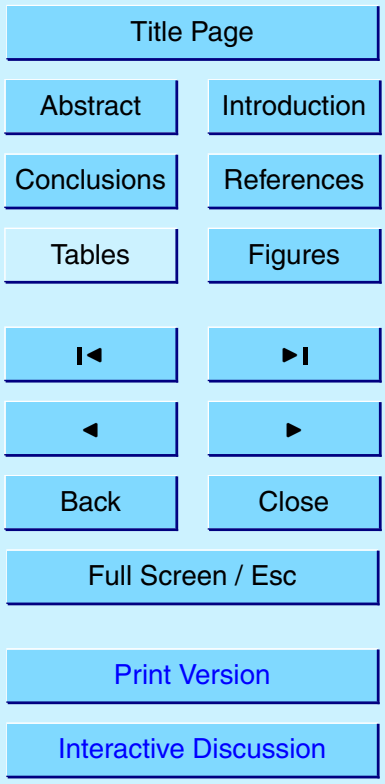

(C) EGU 2003 
temperature $\theta \equiv T_{s}-T_{s 0}\left(T_{s 0}=288 \mathrm{~K}\right)$ :

$p_{s L}=p_{s L 0} \exp \left(\frac{\theta}{\theta_{s}}\right), \theta_{s} \equiv \frac{T_{s 0} T_{s}}{T_{L}}, \quad \theta_{s 0} \equiv \frac{T_{s 0}^{2}}{T_{L}}=18 \mathrm{~K}$.

This means that at $\tau_{S L} \gg 1$ the ratio of the outgoing flux of thermal radiation $F_{L e}$ to the upward flux of thermal radiation at the surface $F_{S L}^{+}$within the absorption interval of water vapor drops exponentially with growing $\theta$ :

$$
\frac{F_{e L}}{F_{s L}^{+}} \propto \frac{1}{\tau_{s L}}=\frac{1}{\tau_{s L 0}} \exp \left(-\frac{\theta}{\theta_{s}}\right) .
$$

In a number of radiative-convective studies where semigrey atmospheres with condensable greenhouse substances were considered, the outgoing flux of thermal radiation into space at high surface temperatures was suggested to remain practically constant (Komabayasi, 1967; Ingersoll, 1969; Kasting, 1988; Goody and Yung, 1989; Nakajima et al., 1992; Pujol and North, 2002). The major assumption responsible for this result is that the entire atmosphere, including the upper radiative layer, is in hydrostatic equilibrium and saturated with respect to water vapor.

The constancy of $F_{L e}$ follows immediately from this assumption. Indeed, in the case of hydrostatic equilibrium the condition $\tau_{L}=1$ (upper radiative layer, where the outgoing flux is formed) unambiguously determines partial pressure of water vapor at this height, which appears to be independent of surface temperature and atmospheric optical thickness, see Eq. (2.4). On the other hand, as far as partial pressure of saturated water vapor is unambiguously related to ambient air temperature in accordance with Clausius-Clapeyron equation (3.1), the temperature $T_{1}$ at the upper radiative layer appears independent of surface temperature either. In the case of constant $T_{1}$ (and assuming local thermodynamic equilibrium), the outgoing thermal radiation into space does not change with growing surface temperature and atmospheric optical thickness.

However, height of convective layer being finite, optical depth at the top of the convective layer $\tau_{c}$ increases with growing $\tau_{s}$. At some value of the latter the upper radiating 6715

\section{Height of convection}

A. M. Makarieva et al.

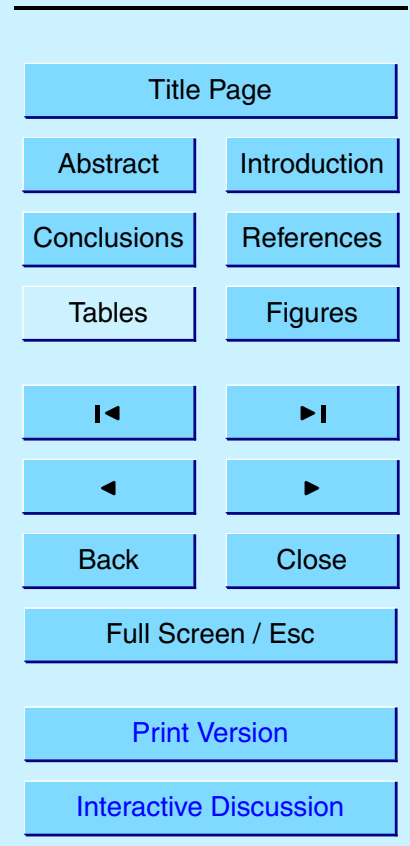

(C) EGU 2003 
layer of the atmosphere $\tau \sim 1$ goes outside the convective layer. There the lapse rate of air temperature at $\tau \sim 1$ drops radically and atmospheric water vapor becomes unsaturated. The dependence between its partial pressure and ambient air temperature, prescribed by Clausius-Clapeyron equation in the case of saturation, vanishes.

5 The fact that the height of convective layer at low latitudes in the modern Earth's climate is close to its maximum value (3.7) testifies that the upper radiative layer corresponding to certain absorption lines of the water vapor absorption interval is located above the convective layer. This suggests that the surface temperatures at which the exponential drop of outgoing radiation is to be observed are not drastically different 10 from those at modern Earth.

\section{Conclusions}

The main result of this paper is that, in contrast to the results obtained in several previous studies (Komabayasi, 1967; Ingersoll, 1969; Kasting, 1988; Goody and Yung, 1989; Nakajima et al., 1992; Rennó, 1997; Pujol and North, 2002), on a planet with 15 an infinite liquid hydrosphere the outgoing flux of thermal radiation into space does not reach a finite plateau with growing surface temperature but diminishes exponentially.

To arrive to this result, we showed that the height of convective layer in an atmosphere with condensable and non-condensable absorbers is finite (in terms of atmospheric scale height $h_{s}$ ) and independent of optical thickness of the atmosphere $\tau_{s}$ at large values of the latter. As far as the dissipative power of the dynamic convective processes is limited by the absorbed solar radiation, the obtained result means that the contribution of convective processes to formation of the outgoing flux of thermal radiation into space tends to zero with increasing atmospheric optical thickness inversely proportionally to the latter. The radiative equilibrium heat transfer described by Eddington approximation becomes the dominant form of heat transfer in the entire atmosphere including the troposphere.

We obtained the exact numerical value of the height of convective layer at the con-

ACPD

3, 6701-6720, 2003

Height of convection

A. M. Makarieva et al.

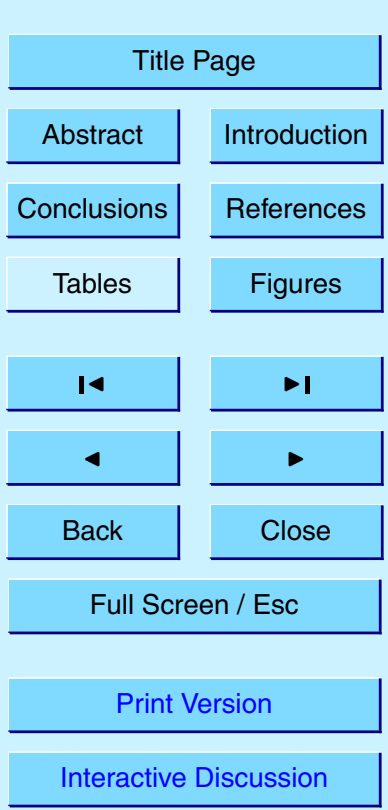

(C) EGU 2003 
dition of large values of optical thickness $\tau_{s}$, which applies to Venus and the equatorial region of Earth (for some part of thermal spectrum). We also showed that at large values of atmospheric optical thickness water vapor at the upper radiative layer will be unsaturated.

5 The assumption of saturation of water vapor in the entire atmosphere, including upper radiative layer, which was used in several radiative-convective studies (Komabayasi, 1967; Ingersoll, 1969; Kasting, 1988; Goody and Yung, 1989; Nakajima et al., 1992; Pujol and North, 2002), may not be used therefore for calculation of the outgoing flux of thermal radiation into space.

10 In the presence of an infinite liquid hydrosphere the outgoing thermal radiation into space should decrease exponentially with growing surface temperature. This is a consequence of an exponential growth with increasing surface temperature of the amount of water in the atmosphere, including cloudiness absorbing thermal radiation over entire thermal spectrum. We conclude that the account of convective processes does not alter this basic physical mechanism of climate change on a planet with liquid hydrosphere.

\section{References}

Allen, C. W.: Astrophysical Quantities, Athlone Press, London, 1973.

Chandrasekhar, S.: An Introduction in the Study of Stellar Structure, Dover, New York, 1957.

20 Goody, R. M. and Yung, Y. L.: Atmospheric radiation, theoretical basis, 2nd edn., Oxford Univ. Press, New York, 1989.

Gorshkov, V. G. and Makarieva, A. M.: Greenhouse effect dependence on atmospheric concentrations of greenhouse substances and the nature of climate stability on Earth, Atmos. Chem. Phys. Discuss., 2, 289-337, 2002.

gersoll, A. P.: The runaway greenhouse: A history of water on Venus, J. Atmos. Sci., 26, 1191-1198, 1969.

Kasting, J. F.: Runaway and moist greenhouse atmospheres and the evolution of Earth and Venus, Icarus, 74, 472-494, 1988.

ACPD

$3,6701-6720,2003$

Height of convection

A. M. Makarieva et al.

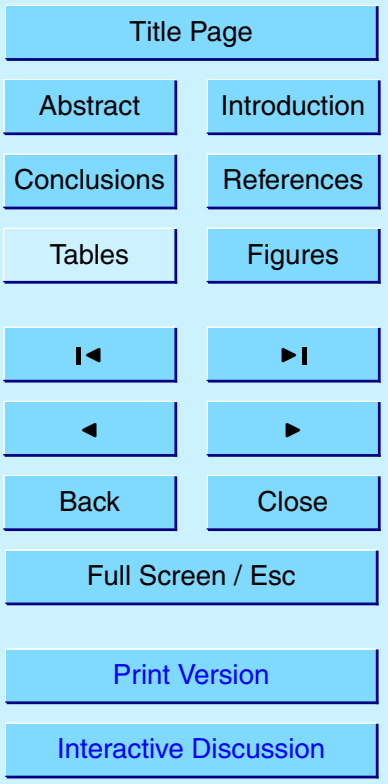

(C) EGU 2003 
Komabayasi, M.: Discrete equilibrium temperatures of a hypothetical planet with the atmosphere and the hydrosphere of one component-two phase system under constant solar radiation, J. Meteorol. Soc. Japan 45, 137-138, 1967.

Michalas, D. and Michalas, B. W.: Foundations of radiation hydrodynamics, Oxford Univ. Press, New York, 1984.

Mitchell, J.: The "greenhouse" effect and climate change, Rev. Geophys., 27, 115-139, 1989.

Nakajima, S., Hayashi, Y. -Y. and Abe, Y.: A study on the "runaway greenhouse effect" with a one-dimensional radiative-convective equilibrium model, J. Atmos. Sci., 49, 2256-2266, 1992.

10 Pujol, T. and North, G. R.: Runaway greenhouse effect in a semigray radiative-convective model, J. Atm. Sci., 59, 2801-2810, 2002.

Ramanathan, V. and Coakley J. A.: Climate modeling through radiative-convective models, Rev. Geophys. Space Phys., 16, 465-489, 1978.

Raval, A. and Ramanathan, V.: Observational determination of the greenhouse effect, Nature, $15 \quad 342,758-761,1989$.

Rennó, N. O.: Multiple equilibria in radiative-convective atmospheres, Tellus 49A, 423-438, 1997.

Weaver, C. P. and Ramanathan, V.: Deductions from a simple climate model: Factors governing surface temperature and atmospheric thermal structure, J. Geophys. Res., 100D, 1158511591, 1995.

ACPD

$3,6701-6720,2003$

Height of convection

A. M. Makarieva et al.

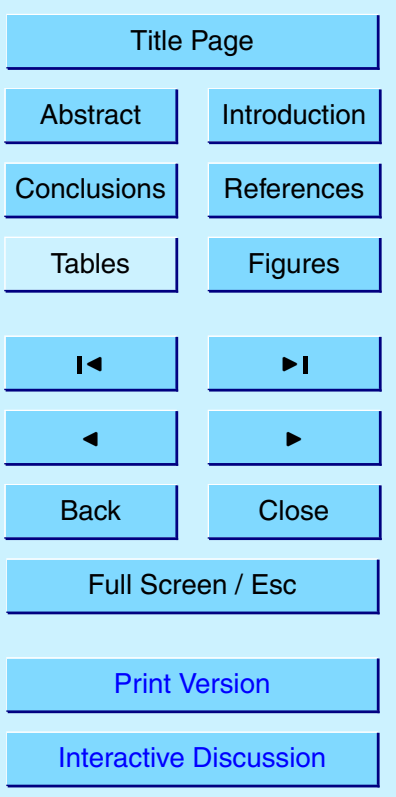

(C) EGU 2003 


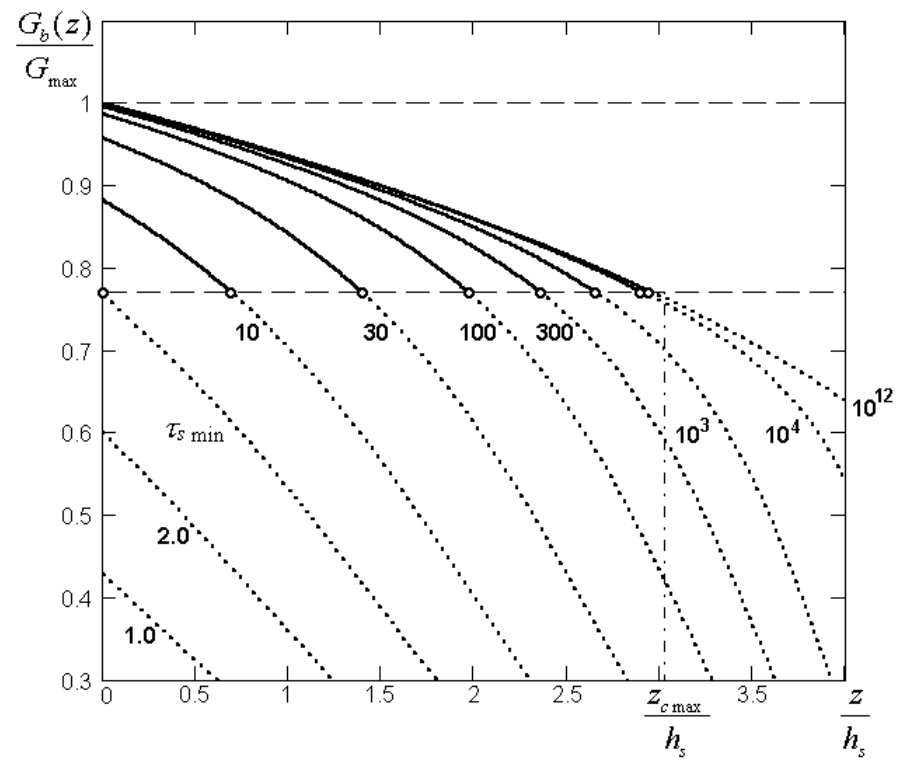

Fig. 1. Lapse rate of brightness temperature $G_{b}$ versus height $z$ for different values of atmospheric optical thickness $\tau_{s}$ of non-condensable greenhouse substances, see Eqs. (2.5) and (2.6). The corresponding values of $\tau_{s}$ are shown below each curve; $h_{s} \equiv R T_{s} / \mathrm{Mg}$. The upper dashed line denotes maximum lapse rate $G_{\max }=M g / 4 R$, the lower dashed line denotes the observed modern lapse rate of air temperature within the convective layer on Earth, $\Gamma=0.77 G_{\max }=6.5 \mathrm{~K} \mathrm{~km}^{-1}$. The height $z_{c}$ of the convective layer for different values of $\tau_{s}$ is determined by the points of intersection (empty circles) of the curves $G_{b}(z)$ with the straight line $\Gamma=0.77 G_{\max }$, see Eq. (2.8). The value of $z_{c \max }=3.03 h_{s}$ (shown by the vertical dash-dotted line), see Eq. (2.11), corresponds to the maximum height of the convective layer observed at $\tau \gg 1$. As far as Eq. (2.6) is valid at $z \leq z_{c}$, parts of curves $G_{b}(z)$ at $z>z_{c}$ are denoted by dots. The onset of convection in the atmosphere occurs at $\tau_{s}=\tau_{s \min }=4.5$, for which $z_{c}=0$. This figure is invariant with respect to changes of surface temperature $T_{s}$.

\section{ACPD}

$3,6701-6720,2003$

Height of convection

A. M. Makarieva et al.

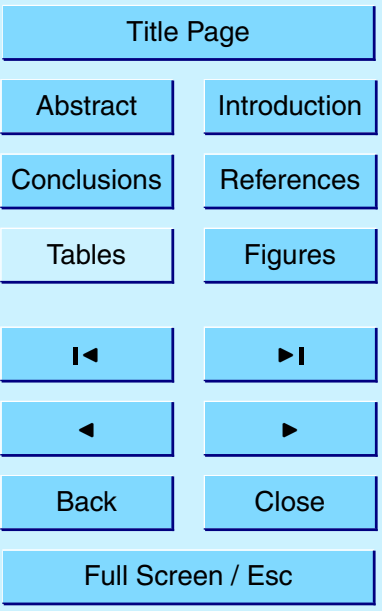

Print Version

Interactive Discussion

(C) EGU 2003 


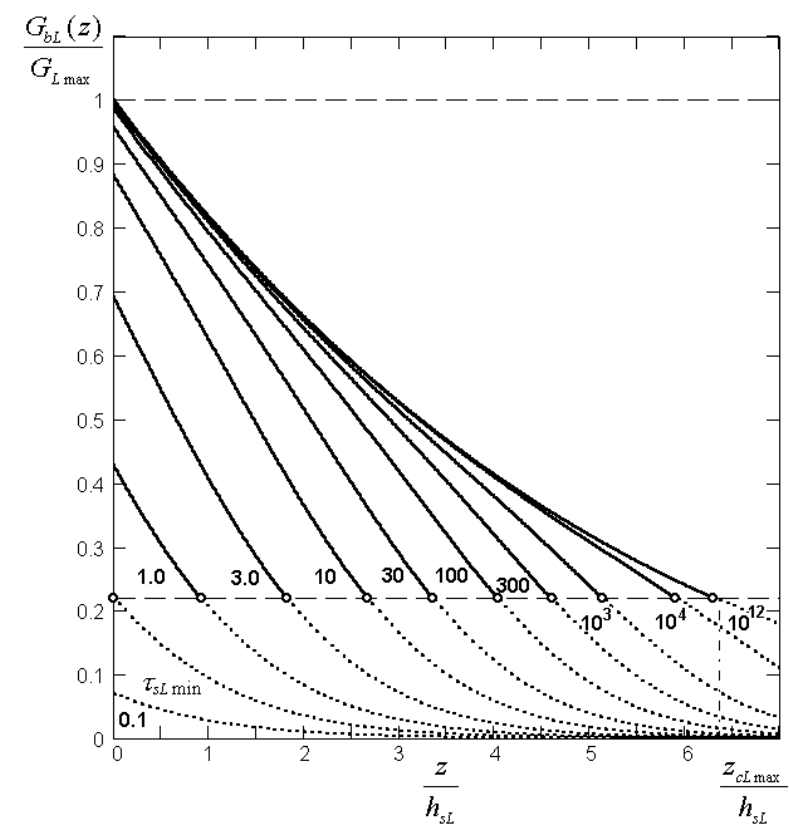

Fig. 2. Lapse rate of brightness temperature $G_{b L}$ versus height $z$ for different values of atmospheric optical thickness $\tau_{s L}$ of water vapor, see Eq. (3.7). The corresponding values of $\tau_{s L}$ are shown below each curve; scale height $h_{s L}=2.4 \mathrm{~km}$. The upper dashed line denotes maximum lapse rate $G_{L \text { max }}=G_{\max } \beta=30 \mathrm{~K} \mathrm{~km}^{-1}, \beta=3.5$; the lower dashed line denotes the observed modern lapse rate of air temperature within the convective layer on Earth, $\Gamma=\alpha_{L} G_{L \text { max }}=6.5 \mathrm{~K} \mathrm{~km}^{-1}, \alpha_{L}=0.22$. Height $z_{C L}$ of the convective layer for different values of $\tau_{s L}$ is determined by the points of intersection of the curves $G_{b L}(z)$ with the straight line $\Gamma=\alpha_{L} G_{L \text { max }}$ (denoted as empty circles). The value of $z_{C L \text { max }}=6.3 h_{s L}$ (shown by the vertical dash-dotted line), see Eq. (3.7), corresponds to the maximum height of the convective layer observed at $\tau_{s L} \gg 1$ and $T_{s}=288 \mathrm{~K}$. As far as Eq. (2.6) is valid within the convective layer, parts of curves $G_{b L}(z)$ at $z>z_{c L}$ are denoted by dots. The onset of convection in the atmosphere occurs at $\tau_{s L}=\tau_{s L \text { min }}=0.38$, for which $z_{c L}=0$.
ACPD

$3,6701-6720,2003$

Height of convection

A. M. Makarieva et al.

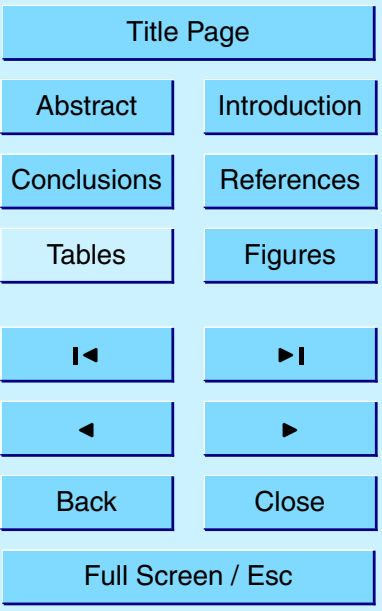

Print Version

Interactive Discussion

(C) EGU 2003 